\title{
An Observational Test of Two-field Inflation
}

\author{
David Wands ${ }^{1}$, Nicola Bartolo ${ }^{2,3,4}$, Sabino Matarrese $e^{2,3}$ and Antonio Riotto ${ }^{3}$ \\ (1) Institute of Cosmology and Gravitation, University of Portsmouth, Portsmouth PO1 2EG, U. K. \\ (2) Dipartimento di Fisica di Padova "G. Galilei", Via Marzolo 8, Padova I-35131, Italy \\ (3) INFN, Sezione di Padova, Via Marzolo 8, Padova I-35131, Italy \\ (4) Astronomy Centre, University of Sussex, Brighton, BN1 9QJ, U. K.
}

(February 1, 2008)

\begin{abstract}
We study adiabatic and isocurvature perturbation spectra produced by a period of cosmological inflation driven by two scalar fields. We show that there exists a model-independent consistency condition for all two-field models of slow-roll inflation, despite allowing for model-dependent linear processing of curvature and isocurvature perturbations during and after inflation on super-horizon scales. The scale-dependence of all spectra are determined solely in terms of slow-roll parameters during inflation and the dimensionless cross-correlation between curvature and isocurvature perturbations. We present additional model-dependent consistency relations that may be derived in specific two-field models, such as the curvaton scenario.
\end{abstract}

PACS numbers: $98.80 . \mathrm{Cq}$

Preprint PU-ICG/02-06, astro-ph/0205253 v2

\section{INTRODUCTION}

The primary interest in inflation [1, 2] is as a mechanism to explain the origin of structure in the Universe from vacuum fluctuations in an early inflationary era which are swept up to arbitrarily large scales. The simplest inflationary models predict an almost scaleinvariant spectrum of Gaussian, adiabatic density perturbations. Such a spectrum was already known as a likely model of structure formation long before inflation was proposed. Inflation also predicts a spectrum of gravitational waves or 'tensor' fluctuations. In single-field models of slow-roll inflation there is a consistency condition between the slope of the spectrum of tensor perturbations and the ratio of tensor to scalar metric fluctuations [1]. The observational confirmation of such a prediction is one of the holy grails of modern cosmology.

Recent studies of multi-field models of inflation have however - threatened to destroy this appealing theoretical prediction. Indeed, entropy perturbations generated in additional light fields can alter the evolution of the curvature perturbation even on large scales. This additional source for the late-time scalar curvature perturbation breaks the single-field consistency relations, yielding only an upper bound on the tensor-scalar ratio [- 6 .

Consistency relations have recently been derived for adiabatic and entropy perturbations during two-field inflation [7]. One of these relations explicitly shows how the single-field consistency condition is modified through the cross-correlation between the entropy and the curvature perturbations. On the other hand, the model dependent nature of reheating at the end of inflation makes it impossible to quantify the late-time amplitude of entropy perturbations solely in terms of the evolution during inflation.

In this paper we introduce a model-independent description of the coupling between adiabatic and entropy perturbations both during and after inflation in order to relate late-time observables to perturbation spectra generated during inflation. We show that the scale dependence of all spectra depend only on quantities at horizon-crossing during slow-roll inflation and the crosscorrelation between curvature and isocurvature perturbations. This cross-correlation generically arises in inflation models for the origin of curvature and isocurvature perturbations [8.9.7. If it can be determined observationally 10 13 then one can reconstruct the scalar curvature perturbation at horizon-crossing. We are thus able to derive a generalised consistency condition for the observed tensor-scalar ratio that holds in all two-field models of slow-roll inflation.

The paper is organized as follows. In section II we introduce the notion of transfer functions and deal with slow-roll inflation in section III. In sections IV and V we compute the power spectra at horizon crossing and at late times, respectively, and derive the consistency relation holding for all two-field models of inflation. In section VI we present additional relations that hold for restricted classes of slow-roll models. Finally, in section VII we draw our conclusions.

\section{TRANSFER FUNCTIONS}

We will first construct dimensionless quantities to describe the instantaneous adiabatic (curvature) and entropy (isocurvature) perturbations both during and after inflation. We can then relate their values on large scales during and after inflation by a model-dependent transfer matrix, whose general form will be given in Eq. (6).

During the conventional radiation-dominated era (after inflation) there is a conventional gauge-invariant definition for the large-scale density/curvature perturbation 14 16] 


$$
\mathcal{R}=\psi+\frac{H \delta \rho}{\dot{\rho}}
$$

where $\psi$ is the gauge-dependent curvature perturbation and $\delta \rho$ the total density perturbation in that gauge. The isocurvature/entropy perturbation is usually defined as the perturbation in the ratio of the matter and photon number densities

$$
\mathcal{S}=\frac{\delta n_{m}}{n_{m}}-\frac{\delta n_{\gamma}}{n_{\gamma}}=-3\left(\frac{H \delta \rho_{m}}{\dot{\rho}_{m}}-\frac{H \delta \rho_{\gamma}}{\dot{\rho}_{\gamma}}\right),
$$

which is naturally gauge-independent.

The 'primordial' adiabatic and isocurvature perturbations on cosmological scales $\left(1-10^{4} \mathrm{Mpc}\right)$ are usually defined in terms of the early-time/large-scale limit deep in the radiation dominated era 17, e.g., around the epoch of primordial nucleosynthesis $\left(T \sim 10^{10} \mathrm{~K}\right)$. The power spectrum and cross-correlation of the primordial adiabatic and isocurvature perturbations on cosmological scales can then be constrained by observations, such as the cosmic microwave background angular power spectrum 18.10 13.

During two-field inflation the general definition of the curvature perturbation (11) yields

$$
\mathcal{R} \simeq \frac{H(\dot{\phi} \delta \phi+\dot{\chi} \delta \chi)}{\dot{\phi}^{2}+\dot{\chi}^{2}} .
$$

where $\simeq$ denotes equality in the slow-roll approximation 7 . The generalised entropy (isocurvature) perturbation is given by [6. 9]

$$
\mathcal{S}=\frac{H(\dot{\phi} \delta \chi-\dot{\chi} \delta \phi)}{\dot{\phi}^{2}+\dot{\chi}^{2}} .
$$

As $\mathcal{S}$ is not directly observable during inflation, its normalisation is somewhat arbitrary. This particular choice keeps the subsequent analysis of power spectra simpler by giving curvature and isocurvature spectra equal power at horizon-crossing [see. Eq. (27)]. A different choice for the normalisation of $\mathcal{S}$ would lead to a different overall factor multiplying the transfer functions $T_{\mathcal{R} S}$ and $T_{\mathcal{S} \mathcal{S}}$ in Eq. (6).

In order to relate the initial curvature and entropy perturbations (3) and (14) generated by a period of inflation in the very early universe, to the observable curvature and entropy perturbations (11) and (2) at much later cosmic times, we need to model the evolution on large ("super-horizon") scales. We will work in a large-scale

\footnotetext{
*Although the curvature and field perturbations are, in general, gauge-dependent, this gauge-dependence can for most purposes be neglected at leading order in the slow-roll approximation. For definiteness one can take all field perturbations to be evaluated in the spatially-flat gauge 19 .
}

limit where the divergence of the velocity field and shear can be neglected so that the local dynamics are those of a homogeneous and isotropic FRW model 16. During slow-roll inflation this becomes a good approximation soon after a mode leaves the Hubble-scale $(k<a H)$, and it remains valid up until the mode re-enters the Hubblescale during the subsequent radiation or matter dominated eras. Adiabatic perturbations correspond to perturbations which locally follow the same trajectory in phase-space as the unperturbed background, whereas entropy perturbations correspond to perturbations off the background trajectory [16].

The curvature perturbation $\mathcal{R}$ remains constant for purely adiabatic perturbations in the large-scale limit simply as a consequence of local energy conservation [16], irrespective of the physical processes going on during inflation or reheating. Purely adiabatic perturbations can never generate entropy perturbations on large scales, but entropy perturbations (specifically a non-adiabatic pressure perturbation or energy transfer) can change the curvature perturbation. Moreover, the entropy perturbation itself can evolve on large scales for imperfect fluidsi . One can thus argue on very general grounds [16] that the time dependence of adiabatic and entropy perturbations in the large-scale limit can always be described by

$$
\dot{\mathcal{R}}=\alpha H \mathcal{S}, \quad \dot{\mathcal{S}}=\beta H \mathcal{S},
$$

where $\alpha$ and $\beta$ are in general time-dependent dimensionless functions. The explicit form of the interaction between the curvature and entropy perturbations has recently been explicitly demonstrated in the case of interacting scalar fields [9,20,21] and non-interacting fluids [22].

Integrating Eqs. (5) over time we can obtain the general form of the transfer matrix relating curvature and entropy perturbations generated when a given mode is stretched outside the Hubble scale during inflation $(k=$ $a H$, denoted by an asterisk) to curvature and entropy perturbations at some later time 13]:

$$
\left(\begin{array}{c}
\mathcal{R} \\
\mathcal{S}
\end{array}\right)=\left(\begin{array}{ll}
1 & T_{\mathcal{R S}} \\
0 & T_{\mathcal{S} S}
\end{array}\right)\left(\begin{array}{l}
\mathcal{R} \\
\mathcal{S}
\end{array}\right)_{*},
$$

where

$$
\begin{aligned}
& T_{\mathcal{R S}}\left(t_{*}, t\right)=\int_{t_{*}}^{t} \alpha\left(t^{\prime}\right) T_{\mathcal{S S}}\left(t_{*}, t^{\prime}\right) H\left(t^{\prime}\right) d t^{\prime}, \\
& T_{\mathcal{S S}}\left(t_{*}, t\right)=\exp \left(\int_{t_{*}}^{t} \beta\left(t^{\prime}\right) H\left(t^{\prime}\right) d t^{\prime}\right) .
\end{aligned}
$$

Although the evolution in the large-scale limit is independent of scale (by definition), the transfer functions

\footnotetext{
${ }^{\dagger}$ For the special case of two non-interacting perfect fluids, such as matter and radiation, the isocurvature perturbation defined in Eq. (2) is constant on large scales.
} 
$T_{\mathcal{R S}}$ and $T_{\mathcal{S S}}$ are implicit functions of scale due to their dependence upon $t_{*}(k)$. The scale-dependence of the transfer functions is proportional to

$$
\begin{aligned}
& H_{*}^{-1} \frac{\partial T_{\mathcal{R} \mathcal{S}}}{\partial t_{*}}=-\alpha_{*}-\beta_{*} T_{\mathcal{R S}}, \\
& H_{*}^{-1} \frac{\partial T_{\mathcal{S} \mathcal{S}}}{\partial t_{*}}=-\beta_{*} T_{\mathcal{S} \mathcal{S}} .
\end{aligned}
$$

Thus the scale-dependence of the transfer functions is determined by $\alpha_{*}$ and $\beta_{*}$ which describe the evolution of the curvature and entropy fluctuations at Hubble-exit during inflation. We shall now show how these can be related to the dimensionless slow-roll parameters during inflation.

\section{SLOW-ROLL INFLATION}

We will consider inflation driven by minimally-coupled real scalar fields $\phi$ and $\chi$ with arbitrary potential $V(\phi, \chi)$. Homogeneous scalar fields in a flat FRW cosmology obey the coupled Klein-Gordon equations

$$
\begin{aligned}
& \ddot{\phi}+3 H \dot{\phi}=-V_{\phi}, \\
& \ddot{\chi}+3 H \dot{\chi}=-V_{\chi},
\end{aligned}
$$

where $V_{x}$ denotes $\partial V / \partial x$, subject to the Friedmann constraint

$$
H^{2}=\frac{8 \pi G}{3}\left(\frac{1}{2} \dot{\phi}^{2}+\frac{1}{2} \dot{\chi}^{2}+V\right) .
$$

Fields interact both through their explicit interaction potential and gravitationally.

Inhomogeneous but linear perturbations about the non-linear but homogeneous background solutions obey the perturbed Klein-Gordon equations which can be written as 23.

$$
\begin{aligned}
\ddot{\delta \phi}+3 H \dot{\delta \phi}+ & {\left[\frac{k^{2}}{a^{2}}+V_{\phi \phi}-\frac{8 \pi G}{a^{3}} \frac{d}{d t}\left(\frac{a^{3}}{H} \dot{\phi}^{2}\right)\right] \delta \phi } \\
& =-\left[V_{\phi \chi}-\frac{8 \pi G}{a^{3}} \frac{d}{d t}\left(\frac{a^{3}}{H} \dot{\phi} \dot{\chi}\right)\right] \delta \chi, \\
\ddot{\delta \chi}+3 H \dot{\delta \chi}+ & {\left[\frac{k^{2}}{a^{2}}+V_{\chi \chi}-\frac{8 \pi G}{a^{3}} \frac{d}{d t}\left(\frac{a^{3}}{H} \dot{\chi}^{2}\right)\right] \delta \chi } \\
& =-\left[V_{\chi \phi}-\frac{8 \pi G}{a^{3}} \frac{d}{d t}\left(\frac{a^{3}}{H} \dot{\chi} \dot{\phi}\right)\right] \delta \phi .
\end{aligned}
$$

where the field perturbations are defined in the spatiallyflat gauge $19,24,25]$.

In the standard approach 5 ,7] one defines five slowroll parameters, two describing the slope of the potential

$$
\epsilon_{\phi} \equiv \frac{1}{16 \pi G}\left(\frac{V_{\phi}}{V}\right)^{2}, \quad \epsilon_{\chi} \equiv \frac{1}{16 \pi G}\left(\frac{V_{\chi}}{V}\right)^{2},
$$

and three describing the curvature

$$
\begin{gathered}
\eta_{\phi \phi} \equiv \frac{1}{8 \pi G}\left(\frac{V_{\phi \phi}}{V}\right), \quad \eta_{\chi \chi} \equiv \frac{1}{8 \pi G}\left(\frac{V_{\chi \chi}}{V}\right), \\
\eta_{\phi \chi} \equiv \frac{1}{8 \pi G}\left(\frac{V_{\phi \chi}}{V}\right) .
\end{gathered}
$$

The slow-roll equations give an approximate solution for the growing mode solution when $\max \left\{\epsilon_{i},\left|\eta_{i j}\right|\right\} \ll 1$. The slow-roll solutions for the homogeneous background are given by

$$
\dot{\phi}^{2} \simeq \frac{2}{3} \epsilon_{\phi} V, \quad \dot{\chi}^{2} \simeq \frac{2}{3} \epsilon_{\chi} V .
$$

where $\simeq$ denotes equality in the slow-roll approximation while linear perturbations on large-scales $(k \ll a H)$ obey

$$
\begin{aligned}
& H^{-1} \dot{\delta \phi} \simeq\left(2 \epsilon_{\phi}-\eta_{\phi \phi}\right) \delta \phi+\left( \pm 2 \sqrt{\epsilon_{\phi} \epsilon_{\chi}}-\eta_{\phi \chi}\right) \delta \chi, \\
& H^{-1} \dot{\delta \chi} \simeq\left(2 \epsilon_{\chi}-\eta_{\chi \chi}\right) \delta \chi+\left( \pm 2 \sqrt{\epsilon_{\chi} \epsilon_{\phi}}-\eta_{\phi \chi}\right) \delta \phi .
\end{aligned}
$$

The $\eta_{i j}$ slow-roll parameters represent the explicit interaction via the potential $V(\phi, \chi)$, while $\epsilon_{i}$ terms are due to the gravitational coupling.

We will adopt the approach of Gordon et al. [9] and perform a local field rotation to identify the instantaneous adiabatic and entropy perturbations along and orthogonal to the background trajectory in field-space:

$$
\begin{gathered}
\delta \sigma=\cos \theta \delta \phi+\sin \theta \delta \chi, \\
\delta s=-\sin \theta \delta \phi+\cos \theta \delta \chi,
\end{gathered}
$$

where $\tan \theta=\dot{\chi} / \dot{\phi} \simeq \pm \sqrt{\epsilon_{\chi} / \epsilon_{\phi}}$. This approach can be readily extended to include multiple scalar fields and nonminimal coupling [21]. The curvature and entropy perturbations, defined in Eqs. (3) and (14) during inflation, then take the simple form

$$
\mathcal{R} \simeq \frac{H \delta \sigma}{\dot{\sigma}}, \quad \mathcal{S}=\frac{H \delta s}{\dot{\sigma}}
$$

The local field rotation (16) allows one of the slowroll parameters to be eliminated, in this case the slope orthogonal to the trajectory, $\epsilon_{s} \simeq 0$ in slow-roll, and we are left with four parameters: one describing the slope of the potential

$$
\epsilon \equiv \frac{1}{16 \pi G}\left(\frac{V_{\sigma}}{V}\right)^{2} \simeq \epsilon_{\phi}+\epsilon_{\chi},
$$

and three describing the curvature

$$
\begin{aligned}
\eta_{\sigma \sigma} & =\eta_{\phi \phi} \cos ^{2} \theta+2 \eta_{\phi \chi} \cos \theta \sin \theta+\eta_{\chi \chi} \sin ^{2} \theta, \\
\eta_{\sigma s} & =\left(\eta_{\chi \chi}-\eta_{\phi \phi}\right) \sin \theta \cos \theta+\eta_{\phi \chi}\left(\cos ^{2} \theta-\sin ^{2} \theta\right), \\
\eta_{s s} & =\eta_{\phi \phi} \sin ^{2} \theta-2 \eta_{\phi \chi} \sin \theta \cos \theta+\eta_{\chi \chi} \cos ^{2} \theta .
\end{aligned}
$$

Alternatively we could choose to diagonalise the mass matrix, $V_{i j}$, to eliminate one $\eta$-term and have two slopes and two curvature parameters. Either way we see that the local evolution of the fields and their perturbations at any instant can be described by four slow-roll parameters. 
The background slow-roll solution is then given by

$$
\dot{\sigma}^{2} \simeq \frac{2}{3} \epsilon V, \quad H^{-1} \dot{\theta} \simeq-\eta_{\sigma s},
$$

while the perturbations obey

$$
\begin{aligned}
H^{-1} \dot{\delta \sigma} & \simeq\left(2 \epsilon-\eta_{\sigma \sigma}\right) \delta \sigma-2 \eta_{\sigma s} \delta s, \\
H^{-1} \dot{\delta} s & \simeq-\eta_{s s} \delta s .
\end{aligned}
$$

The entropy field perturbation $\delta s$ evolves independently of the adiabatic field perturbation $\delta \sigma$ on large scales. However the large-scale entropy perturbations do affect the evolution of the adiabatic perturbations when $\eta_{\sigma s} \neq 0$.

In terms of the dimensionless perturbations $\mathcal{R}$ and $\mathcal{S}$ we have

$$
\begin{aligned}
\dot{\mathcal{R}} & \simeq-2 \eta_{\sigma s} H \mathcal{S}, \\
\dot{\mathcal{S}} & \simeq\left(-2 \epsilon+\eta_{\sigma \sigma}-\eta_{s s}\right) H \mathcal{S} .
\end{aligned}
$$

which provides a specific example of the more general form for the evolution of curvature and entropy perturbations given in Eqs. (5). In particular the scaledependence of the integrated transfer functions, Eqs. (8), can be written in terms of the slow-roll parameters when the mode crosses outside the Hubble-scale:

$$
\begin{aligned}
& \alpha_{*} \simeq-2 \eta_{\sigma s}, \\
& \beta_{*} \simeq-2 \epsilon+\eta_{\sigma \sigma}-\eta_{s s} .
\end{aligned}
$$

\section{INITIAL POWER SPECTRA}

Weakly-interacting, light fields acquire a spectrum of vacuum fluctuations at Hubble-crossing $\left(k=a_{*} H_{*}\right)$ [1]

$$
\left.\left.\mathcal{P}_{\delta \phi}\right|_{*} \simeq \mathcal{P}_{\delta \chi}\right|_{*} \simeq\left(\frac{H_{*}}{2 \pi}\right)^{2},
$$

which describe independent Gaussian random fields, i.e. zero cross-correlation

$$
\left.\mathcal{C}_{\delta \phi, \delta \chi}\right|_{*}=0 .
$$

The local rotation (16) to the instantaneous adiabatic and entropy field perturbations, gives

$$
\begin{gathered}
\left.\left.\mathcal{P}_{\delta \sigma}\right|_{*} \simeq \mathcal{P}_{\delta s}\right|_{*} \simeq\left(\frac{H_{*}}{2 \pi}\right)^{2}, \\
\left.\mathcal{C}_{\delta \sigma, \delta s}\right|_{*}=0 .
\end{gathered}
$$

Hence, using Eq. (17), the adiabatic and entropy power spectra at Hubble-crossing are given by

$$
\left.\left.\mathcal{P}_{\mathcal{R}}\right|_{*} \simeq \mathcal{P}_{\mathcal{S}}\right|_{*} \simeq\left(\frac{H^{2}}{2 \pi \dot{\sigma}}\right)_{*}^{2} \simeq \frac{8}{3 \epsilon} \frac{V_{*}}{M_{P}^{4}} .
$$

Although, as explained earlier, the normalisation of the dimensionless entropy perturbation during inflation is arbitrary, it proves convenient to use that given in Eq. (4) so that $\mathcal{R}$ and $\mathcal{S}$ have equal power at Hubble-crossing.

The spectral tilts (defined by $n_{x} \equiv d \ln \mathcal{P}_{x} / d \ln k$ ) are given by

$$
\left.\left.n_{\mathcal{R}}\right|_{*} \simeq n_{\mathcal{S}}\right|_{*} \simeq-6 \epsilon+2 \eta_{\sigma \sigma} .
$$

Gravitational waves are generated with a spectrum [1]

$$
\left.\mathcal{P}_{T}\right|_{*} \simeq \frac{128}{3} \frac{V_{*}}{M_{P}^{4}}
$$

and spectral tilt

$$
\left.n_{T}\right|_{*} \simeq-2 \epsilon .
$$

A key observation is that the tensor-scalar ratio at Hubble-crossing, even in multi-field slow-roll inflation, can be given from Eqs. (27), (29) and (30) as

$$
\left(\frac{\mathcal{P}_{T}}{\mathcal{P}_{\mathcal{R}}}\right)_{*} \simeq 16 \epsilon \simeq-\left.8 n_{T}\right|_{*} .
$$

\section{FINAL POWER SPECTRA}

Applying the transfer matrix (5) to the initial scalar spectra we obtain the resulting curvature and isocurvature power spectra at the start of the conventional radiation-dominated era:

$$
\begin{aligned}
\mathcal{P}_{\mathcal{R}} & =\left.\left(1+T_{\mathcal{R} \mathcal{S}}^{2}\right) \mathcal{P}_{\mathcal{R}}\right|_{*}, \\
\mathcal{P}_{\mathcal{S}} & =\left.T_{\mathcal{S} \mathcal{S}}^{2} \mathcal{P}_{\mathcal{R}}\right|_{*}, \\
\mathcal{C}_{\mathcal{R S}} & =\left.T_{\mathcal{R S}} T_{\mathcal{S} \mathcal{S}} \mathcal{P}_{\mathcal{R}}\right|_{*} .
\end{aligned}
$$

A dimensionless measure of the correlation can be defined in terms of a correlation angle $\Delta$ such that

$$
\cos \Delta \equiv \frac{\mathcal{C}_{\mathcal{R S}}}{\mathcal{P}_{\mathcal{R}}^{1 / 2} \mathcal{P}_{\mathcal{S}}^{1 / 2}} \simeq \frac{T_{\mathcal{R S}}}{\sqrt{1+T_{\mathcal{R S}}^{2}}}
$$

Note that the scalar metric perturbation at Hubblecrossing can thus be reconstructed from the observed curvature perturbation at late times and the crosscorrelation angle:

$$
\left.\mathcal{P}_{\mathcal{R}}\right|_{*} \simeq \mathcal{P}_{\mathcal{R}} \sin ^{2} \Delta .
$$

The tensor perturbations, in contrast to the scalar perturbations, remain 'frozen-in' on large scales, and decoupled from the scalar perturbations at linear order. Thus the primordial perturbation spectrum for gravitational waves is given by Eqs. (29) and (30)

$$
\mathcal{P}_{T}=\left.\mathcal{P}_{T}\right|_{*}, \quad n_{T}=\left.n_{T}\right|_{*} .
$$


The consistency condition for the tensor-scalar amplitudes at Hubble-crossing can thus be rewritten using Eqs. (31), (36) and (37) as a consistency relation between the tensor-scalar amplitudes at late times:

$$
\frac{\mathcal{P}_{T}}{\mathcal{P}_{\mathcal{R}}} \simeq-8 n_{T} \sin ^{2} \Delta .
$$

Any two-field model of slow-roll inflation predicts that this relation should hold between quantities which are directly observable, so long as the amplitude of the isocurvature and tensor perturbations prove to be large enough. This relation was first obtained in Ref. [7] just at the end of an inflationary period where two scalar fields are present (see Eq. (59) of Ref. [7]). We have shown that this consistency relation (38) between the tensor-scalar amplitudes is not modified by any linear processes the matter perturbations may undergo between the end of inflation and some later time $t$.

The scale-dependence of the final scalar power spectra depends both on the scale dependence of the initial spec$\operatorname{tra}\left(\left.n_{\mathcal{R}}\right|_{*}\right)$ and on the transfer functions $T_{\mathcal{R} S}$ and $T_{\mathcal{S} S}$. The spectral tilts are given from Eqs. (32 34) by

$$
\begin{aligned}
n_{\mathcal{R}} & =\left.n_{\mathcal{R}}\right|_{*}+H_{*}^{-1}\left(\partial T_{\mathcal{R S}} / \partial t_{*}\right) \sin 2 \Delta, \\
n_{\mathcal{S}} & =\left.n_{\mathcal{R}}\right|_{*}+2 H_{*}^{-1}\left(\partial \ln T_{\mathcal{S} \mathcal{S}} / \partial t_{*}\right), \\
n_{\mathcal{C}} & =\left.n_{\mathcal{R}}\right|_{*}+H_{*}^{-1}\left[\left(\partial T_{\mathcal{R} \mathcal{S}} / \partial t_{*}\right) \tan \Delta+\left(\partial \ln T_{\mathcal{S S}} / \partial t_{*}\right)\right],
\end{aligned}
$$

where we have used Eq. (35) to eliminate $T_{\mathcal{R S}}$ in favour of the observable correlation angle $\Delta$. Substituting in Eq. (28) for the tilt at Hubble-exit, and Eqs. (8) and (23) for the scale-dependence of the transfer functions, we obtain

$$
\begin{aligned}
n_{\mathcal{R}} \simeq & -\left(6-4 \cos ^{2} \Delta\right) \epsilon \\
& +2\left(\eta_{\sigma \sigma} \sin ^{2} \Delta+2 \eta_{\sigma s} \sin \Delta \cos \Delta+\eta_{s s} \cos ^{2} \Delta\right) \\
n_{\mathcal{S}} \simeq & -2 \epsilon+2 \eta_{s s}, \\
n_{\mathcal{C}} \simeq & -2 \epsilon+2 \eta_{s s}+2 \eta_{\sigma s} \tan \Delta .
\end{aligned}
$$

We emphasize that although the overall amplitude of the transfer functions $T_{\mathcal{R} S}$ and $T_{\mathcal{S S}}$ are dependent upon the evolution after Hubble-exit, through reheating and into the radiation dominated era, the spectral tilts of the resulting perturbation spectra can be expressed solely in terms of the slow-roll parameters at Hubble-crossing during inflation and the correlation angle $\Delta$ which can (in principle) be determined by observations.

\footnotetext{
${ }^{\ddagger}$ In this notation, the spectral index for adiabatic scalar perturbations is conventionally given as $n=1+n_{\mathcal{R}}$ so that a scale-invariant (Harrison-Zel'dovich) spectrum corresponds to $n_{\mathcal{R}}=0$.
}

\section{MODEL-DEPENDENT RELATIONS}

In addition to the consistency condition (38) that applies to any slow-roll model of two-field inflation, there are additional model-dependent relations that will hold for restricted classes of two-field inflation.

In Ref. [7] a second consistency relation was derived for curvature and entropy perturbations at the end of inflation using the integrated slow-roll solutions:

$$
n_{\mathcal{C}}-n_{\mathcal{S}}+\frac{n_{\mathcal{R}}+n_{\mathcal{S}}-2 n_{\mathcal{C}}}{2 \sin ^{2} \Delta} \approx 0 .
$$

From Eqs. (39) one sees that in fact this holds for all models for which $H_{*}^{-1} \partial \ln T_{\mathcal{S S}} / \partial t_{*} \approx 0$. This requires $\beta_{*} \approx 0$ in Eq. (8) which can be given as a constraint on the slow-roll parameters at Hubble-exit by Eq. (23).

Another class of two-field inflation models are those in which the curvature and isocurvature perturbations are effectively decoupled around the time of Hubbleexit, $\alpha_{*} \approx 0$ in Eq. (5). In terms of slow-roll parameters at Hubble-exit this requires, from Eq. (23), that $\eta_{\sigma s} \approx 0$. This includes models in which only one scalar field evolves during inflation, but where both fields play a significant dynamical role during reheating or afterwards. From Eqs. (40) we see immediately that we have the constraint

$$
n_{\mathcal{S}} \approx n_{\mathcal{C}}
$$

The curvaton model for the origin of cosmological structure proposed in Ref. 26,27] falls into this class of models. In the curvaton scenario the initial curvature perturbation at Hubble-exit (or later) during inflation is taken to be negligible, i.e., $\mathcal{R}_{*} \ll \mathcal{R}$. The curvature perturbation observed during the subsequent radiation or matter dominated eras is supposed to be due entirely to an initial isocurvature perturbation at Hubble-exit. As can be seen from Eq. (36) this implies that the resulting curvature and isocurvature perturbations must be $100 \%$ correlated, $\sin \Delta \approx 0$. Unfortunately Eq. (38) then shows that the amplitude of tensor perturbations must be negligible, $\mathcal{P}_{T} \approx 0$. Instead we have a constraint solely in terms of the scalar spectra from Eqs. (40):

$$
n_{\mathcal{R}} \approx n_{\mathcal{S}} \approx n_{\mathcal{C}}
$$

\section{CONCLUSIONS}

In this paper we have shown how curvature and entropy perturbations produced by any slow-roll model of two-field inflation can be related to observable curvature and matter-isocurvature perturbation spectra at late times.

The resulting amplitude and tilt of the spectra of curvature and entropy perturbations and their correlation 
can be described by six parameters which may in principle be determined observationally. These six observables are determined by seven model parameters: the Hubble rate during inflation, four dimensionless slow-roll parameters, and two transfer functions $T_{\mathcal{R S}}$ and $T_{\mathcal{S S}}$ which are dependent upon the detailed physics of reheating. This situation is analogous to the case of single-field inflation where the two observables (amplitude and tilt) of the adiabatic curvature perturbation spectrum is determined by three model parameters: the Hubble rate during inflation and two slow-roll parameters.

To break the degeneracy we require an observable spectrum of gravitational waves produced during inflation, whose amplitude and tilt gives two more observables, and hence the observationally testable consistency relation (38), which is a generalisation of the single-field relation 11.

Although the amplitude of the isocurvature and crosscorrelation spectra are dependent upon two transfer functions $T_{\mathcal{R S}}$ and $T_{\mathcal{S S}}$ which are, a priori, unknowns, the correlation angle, $\Delta$, is a direct measure of one of these, $T_{\mathcal{R S}}$. This enables one to quantify the contribution of non-adiabatic perturbations to the late-time curvature and hence reconstruct the original curvature perturbation spectrum at Hubble-exit from that observed at late times. A measure of the amplitude of the late-time isocurvature amplitude then allows one to determine $T_{\mathcal{S S}}$.

We have shown that the spectral tilts of the tensor and scalar spectra can be written in terms of the four slowroll parameters describing the evolution at the time of Hubble-exit during inflation. This yields additional consistency relations in specific models such as the curvaton scenario [26,27].

Finally we note that the relation (36) between the isocurvature correlation angle $\Delta$ and the change in the large-scale curvature assumes that only one entropy mode exists at horizon-exit during inflation. In inflation models with more than two light fields during inflation, an additional uncorrelated entropy mode at horizon-crossing could contribute to the isocurvature without affecting the curvature at late times. Thus our generalised consistency relation (38) only applies to two-field models of inflation. For three or more light fields during inflation we again have an inequality

$$
\frac{\mathcal{P}_{T}}{\mathcal{P}_{\mathcal{R}}} \leq-8 n_{T} \sin ^{2} \Delta .
$$

At the same time, we have only considered one observable isocurvature mode in the radiation-dominated era. The general cosmological perturbation can include as many isocurvature modes as there are distinguishable matter components [28]. The correlation of these additional modes with the curvature could enable one to reconstruct the curvature perturbation at horizon-exit even in the presence of additional light fields during inflation. In general one would expect to be able to find a tensor-scalar consistency condition when there are as many observable perturbation modes in the radiation dominated universe after inflation as there are light fields during inflation.

\section{ACKNOWLEDGMENTS}

NB was partially supported by a Marie Curie Fellowship of the European Community programme HUMAN POTENTIAL under contract HPMT-CT-200000096. DW is supported by the Royal Society.

[1] A. R. Liddle and D. H. Lyth, "Cosmological Inflation And Large-Scale Structure," Cambridge, UK: Univ. Pr. (2000) $400 \mathrm{p}$.

[2] D. H. Lyth and A. Riotto, Phys. Rept. 314, 1 (1999) arXiv:hep-ph/9807278.

[3] D. Wands, arXiv:astro-ph/0201541.

[4] D. Polarski and A. A. Starobinsky, Phys. Lett. B 356 , 196 (1995) arXiv:astro-ph/9505125.

[5] M. Sasaki and E. D. Stewart, Prog. Theor. Phys. 95, 71 (1996) arXiv:astro-ph/9507001.

[6] J. Garcia-Bellido and D. Wands, Phys. Rev. D 53, 5437 (1996) arXiv:astro-ph/9511029.

[7] N. Bartolo, S. Matarrese and A. Riotto, Phys. Rev. D 64, 123504 (2001) arXiv:astro-ph/0107502.

[8] D. Langlois, Phys. Rev. D 59, 123512 (1999).

[9] C. Gordon, D. Wands, B. A. Bassett and R. Maartens, Phys. Rev. D 63, 023506 (2001) arXiv:astro-ph/0009131.

[10] D. Langlois and A. Riazuelo, Phys. Rev. D 62, 043504 (2000) arXiv:astro-ph/9912497.

[11] M. Bucher, K. Moodley and N. Turok, Phys. Rev. Lett. 87, 191301 (2001) [arXiv:astro-ph/0012141.

[12] R. Trotta, A. Riazuelo and R. Durrer, Phys. Rev. Lett. 87, 231301 (2001) arXiv:astro-ph/0104017.

[13] L. Amendola, C. Gordon, D. Wands and M. Sasaki, arXiv:astro-ph/0107089.

[14] J. M. Bardeen, P. J. Steinhardt and M. S. Turner, Phys. Rev. D 28, 679 (1983).

[15] J. M. Bardeen, DOE/ER/40423-01-C8 Lectures given at 2nd Guo Shou-jing Summer School on Particle Physics and Cosmology, Nanjing, China, Jul 1988.

[16] D. Wands, K. A. Malik, D. H. Lyth and A. R. Liddle, Phys. Rev. D 62, 043527 (2000) arXiv:astro$\mathrm{ph} / 0003278$.

[17] H. Kodama and M. Sasaki, Int. J. Mod. Phys. A 1, 265 (1986); Int. J. Mod. Phys. A 2, 491 (1987); J. R. Bond and G. Efstathiou, Mon. Not. Roy. Astron. Soc. 226, 655 (1987); M. Bucher, K. Moodley and N. Turok, Phys. Rev. D 62, 083508 (2000) arXiv:astro-ph/9904231.

[18] K. Enqvist, H. Kurki-Suonio and J. Valiviita, Phys. Rev. D 65, 043002 (2002) arXiv:astro-ph/0108422.

[19] H. Kodama and M. Sasaki, Prog. Theor. Phys. Suppl. 78, 1 (1984). 
[20] J. c. Hwang and H. Noh, Phys. Lett. B 495, 277 (2000) arXiv:astro-ph/0009268.

[21] S. Groot Nibbelink and B. J. van Tent, Class. Quant. Grav. 19, 613 (2002) arXiv:hep-ph/0107272.

[22] J. c. Hwang and H. Noh, Class. Quant. Grav. 19, 527 (2002) arXiv:astro-ph/0103244.

[23] A. Taruya and Y. Nambu, Phys. Lett. B 428, 37 (1998) arXiv:gr-qc/9709035.

[24] M. Sasaki, Prog. Theor. Phys. 76, 1036 (1986).

[25] J. C. Hwang, Class. Quant. Grav. 11, 2305 (1994).

[26] D. H. Lyth and D. Wands, Phys. Lett. B 524, 5 (2002) arXiv:hep-ph/0110002.

[27] T. Moroi and T. Takahashi, Phys. Lett. B 522, 215 (2001) arXiv:hep-ph/0110096.

[28] M. Bucher, K. Moodley and N. Turok, Phys. Rev. D 62, 083508 (2000) arXiv:astro-ph/9904231. 